\title{
Power law fluctuation generator based on analog electrical circuit
}

\author{
Aki-Hiro Sato ${ }^{1 *}$ Hideki Takayasu ${ }^{2}$ and Yasuji Sawada ${ }^{1}$ \\ ${ }^{1}$ Research Institute of Electrical Communication, \\ Tohoku University, Sendai 980-8577, Japan. \\ 2 Sony Computer Science Lab., Takanawa Muse Bldg., 3-14-13, \\ Higashi-Gotanda, Shinagawa-ku, Tokyo 141-0022, Japan.
}

October 30, 2018

\begin{abstract}
We constructed an analog circuit generating fluctuations of which a probability density function has power law tails. In the circuit fluctuations with an arbitrary exponent of the power law can be obtained by tuning the resistance. A theory of a differential equation with both multiplicative and additive noises which describes the circuit is introduced. The circuit is composed of a noise generator, an analog multiplier and an integral circuit. Sequential outputs of the circuit are observed and their probability density function and autocorrelation coefficients are shown. It is found that correlation time of the autocorrelation coefficient is dependent on the power law exponent.
\end{abstract}

*Electronic mail:aki@sawada.riec.tohoku.ac.jp 


\section{Introduction}

Random multiplicative process (RMP) is attracting much interest recently as a new mechanism of generating power law fluctuations. The process is described by a Langevin system with both multiplicative and additive noises. It was proved that a PDF for its amplitude has power law tails when the noises are independent [1, 2, 3. The continuous time version of RMP is described by a stochastic differential equation with both multiplicative and additive noises. It was shown that a stationary probability density function for its amplitude has power law tails when the noises are Gaussian white noise [4, 5, 6]. This type of equations has been studied in chaos on-off intermittency[7], motions of a polymer in turbulent fluid[8], nonlinear coupled oscillators[9] and price changes in economics [10].

In this paper we propose an electrical generator of random fluctuations based on the theory of RMP for the power law fluctuations by introducing an analog electrical circuit consisted of operational amplifiers and an analog multiplier.

The paper is organized as follows. In Sec. 2 we make a brief explanation of the theory of the continuous time RMP. In Sec. 3 we show the circuit diagram of the proposed generator having power law fluctuations and explain its mechanism. In Sec. \# we show the data of sequential outputs, their probability distribution function (PDF) and their autocorrelation coefficients with discussion. Sec. 5 is devoted for concluding remarks.

\section{Theory of RMP}

We here review a brief outline of the stochastic differential equation with both multiplicative and additive noises 4 , 5, 6]. The stochastic differential equation is given by 
where $v(t)$ is a dynamical variable, and $\nu(t)$ and $\xi(t)$ represent a multiplicative noise and an additive noise, respectively. Representing ensemble average by $\langle\ldots\rangle$ we require $\langle\nu(t)\rangle=\bar{\nu}$, and $\langle\xi(t)\rangle=0$. When $\nu(t)$ and $\xi(t)$ follow Gaussian white noises with their strengths characterized by $D_{\nu}$ and $D_{\xi}$, respectively,

$$
\begin{aligned}
\left\langle\left[\nu\left(t_{1}\right)-\bar{\nu}\right]\left[\nu\left(t_{2}\right)-\bar{\nu}\right]\right\rangle & =2 D_{\nu} \delta\left(t_{1}-t_{2}\right), \\
\left\langle\xi\left(t_{1}\right) \xi\left(t_{2}\right)\right\rangle & =2 D_{\xi} \delta\left(t_{1}-t_{2}\right),
\end{aligned}
$$

the PDF for the dynamical variable $v$ in eq. (1), $p(v, t)$, is known to follow the generalized Fokker-Planck equation,

$$
\frac{\partial}{\partial t} p(v, t)=\frac{\partial}{\partial v}\left[-\left(\bar{\nu}+D_{\nu}\right) v+\frac{\partial}{\partial v}\left(D_{\nu} v^{2}+D_{\xi}\right)\right] p(v, t)
$$

A steady state solution corresponding to a zero-current state can be obtained by putting the term of $\frac{\partial}{\partial v}$ to 0 . By solving eq. (田) we get a stationary distribution,

$$
p(v) \propto\left(D_{\xi}+D_{\nu} v^{2}\right)^{\frac{\bar{\nu}}{2 D_{\nu}}-\frac{1}{2}}
$$

which has power law tails for large $v$,

$$
p(v) \propto|v|^{\bar{\nu} / D_{\nu}-1}
$$

For convenience of notation we define an exponent of the power law tail as

$$
p(v) \propto|v|^{-\beta-1}
$$

Comparing eq. (6) with eq. (团) yields

$$
\beta=-\frac{\bar{\nu}}{D_{\nu}}
$$

It is useful to introduce a cumulative distribution function (CDF) defined as

$$
P(\geq|v|)=\int^{-|v|} p\left(v^{\prime}\right) d v^{\prime}+\int^{\infty} p\left(v^{\prime}\right) d v^{\prime}
$$


Then the CDF of $v$ in eq. (11) is given as

$$
P(\geq|v|) \propto|v|^{-\beta}
$$

namely, the slope in log-log plots directly gives the power law exponent $\beta$. The corresponding PDF of eq. (5) is given as

$$
p(v) \propto\left(1+\frac{v^{2}}{s^{2}}\right)^{-\beta / 2-1 / 2},
$$

where $s=\sqrt{\frac{D_{\xi}}{D_{\nu}}}$. Namely $p(v)$ has power law tails for $v>s$.

\section{The circuit}

From the assumption for the multiplicative noise in the above theory it is important that $\nu(t)$ takes both positive and negative values to realize the power law tails. In an electrical circuit this means that it is necessary for the circuit to include both positive and negative feedbacks. We solve this problem by using an analog multiplier. A block diagram of our circuit and an implemented noise generator are shown in fig.11. In the figures $v_{o}$ represents the output voltage, and $\mu(t)$ is the output directly from the noise generator. As seen from this figure the circuit contains the noise generator, an analog multiplier (Analog devices, 10MHz, 4-quadrant) and an operational amplifier (National Semiconductor, LF157) for integrator.

The output of the noise generator plays a role of the multiplicative noise in eq. (11). In the noise generator a shot noise between the zener diode in fig. 1 is amplified by an operational amplifier, it passes through a high pass filter to be taken out from the circuit. LF157 has the $20 \mathrm{M}$ product of a voltage gain $(\mathrm{G})$ and a bandwidth $(\mathrm{B})$. The bandwidth is given by $\mathrm{B}=200 \mathrm{kHz}$ in the noise generator because we put $G=100$. Thus we expect a frequency characteristics of the noise generator to be up to $200 \mathrm{kHz}$. We perform the positive and negative feedback bv multiplving $v_{0}$ with the output of the noise 
negative input with the operational amplifier for integrator. Although the additive noise term is not explicitly added in the circuit, it derives from either thermal noises of the operational amplifier or from an external electromagnetic noises.

An equation equivalent to the circuit diagram of fig. 11 is given as

$$
\frac{d v_{o}}{d t}=\left(\frac{1}{R_{f} C}+\frac{k}{R_{v} C} \mu(t)\right) v_{o}+\xi(t),
$$

where $k$ is a factor of the multiplier with $k=1 / 10$, and $\xi(t)$ represents the additive noise effect. The strength of multiplicative noise $\mu(t)$ depends on the value of a variable resistor $R_{v}$ in fig. 11 because $R_{v}$ is a factor of $\mu(t)$ in eq. (12). Therefore, we expect that the power law exponent for the output is a function of $R_{v}$ like eq. (8). For the output of the noise generator $\mu(t)$ we show a PDF and an autocorrelation coefficient in figs. 2 and 3, respectively. The autocorrelation coefficient is defined as

$$
K_{1}(\tau)=\frac{\langle\mu(t+\tau) \mu(t)\rangle-\langle\mu(t+\tau)\rangle\langle\mu(t)\rangle}{\left\langle\mu(t)^{2}\right\rangle-\langle\mu(t)\rangle^{2}}
$$

We sampled outputs of the noise generator through 12bit AD-converter (Microscience, ADM-652AT) and processed them in a computer. A sampling frequency is $125 \mathrm{kHz}$. We find that the PDF can be approximated by the Gaussian distribution and that the autocorrelation coefficient decays quickly. This result means that the frequency characteristics of the noise generator is up to around $200 \mathrm{kHz}$. Therefore we consider $\mu(t)$ as a Gaussian white noise under $200 \mathrm{kHz}$.

\section{Results and Discussions}

We observe the output of the circuit $v_{o}$ in respect of $R_{v}$ since we expect that the power law exponent $\beta$ is a function of $R_{v}$ from the theory. We detected the output through a 12-bit AD converter and processed it as digital 
we explain the method of adjusting an offset of the operational amplifier for integrator. We adjust the variable resistor added to the operational amplifier for a temporal average of output $\langle v\rangle$ to be almost likely zero when the value of $R_{v}$ is the smallest. As shown in fig. 4 the reason is that the temporal average shows exponential decay for $R_{v}$. We keep the zero-average of $v(t)$ throughout all observations in this way.

We show examples of temporal output at $R_{v}=5$ and $R_{v}=150$ in fig. 5. We find that amplitude of the output $v(t)$ depends on the value of $R_{v}$. The PDF for the temporal output on $R_{v}=5 \Omega$ and $R_{v}=150 \Omega$ are shown in fig.6. The tail's form of the PDF depends on the value of $R_{v}$. To see the difference between the positive and negative tails in detail we show log-log plots of the PDF in the positive and negative domains in fig. 7. It is obvious that the slope of the tail in the positive domain looks different from that in the negative domain. There are tow possible reasons on this effect. One is that an average of the additive noise is not completely zero. The other is that a distribution of the additive noise is asymmetrical.

To roughly estimate the dependence of the exponent $\beta$ on $R_{v}$ we show $\log -\log$ plots of CDF of $v(t)$ at $R_{v}=5,25,50,100,150 \Omega$ in fig. 8. Log-log plots of CDF have a liner part for about a decade. We clearly find that the exponent $\beta$ depends on $R_{v}$ as expected qualitatively. However, we can not apply eq. (8) directly to these experimental results because the multiplicative noise generated by the noise generator is not an ideal white noise but is correlated at some frequencies. The authors have proved a relation to the power law exponent of RMP with correlated multiplicative noise in a discrete time version [11], yet, in the continuous time version the theoretical relation between the exponent and the correlated multiplicative noise is an interesting open problem. 
eq. (11). Namely, the strength of the additive noise is $10^{-2}$ times as large as that of the multiplicative noise.

The autocorrelation coefficient of outputs, defined in eq. (13), are shown in fig. 9. A correlated time of the autocorrelation coefficient $K_{1}(\tau)$ on $R_{v}=5 \Omega$ is longer than the case of $R_{v}=150 \Omega$. This implies that the correlation time depends on the value of $R_{v}$. In economics it is often more interesting to observe the autocorrelation coefficient of squared outputs than the standard autocorrelation coefficient [12]. The autocorrelation coefficient of squared outputs called the volatility correlation is defined as

$$
K_{2}(\tau)=\frac{\left\langle v(t+\tau)^{2} v(t)^{2}\right\rangle-\left\langle v(t+\tau)^{2}\right\rangle\left\langle v(t)^{2}\right\rangle}{\left\langle v(t)^{4}\right\rangle-\left\langle v(t)^{2}\right\rangle^{2}} .
$$

As shown in fig. 10 the correlation time of the autocorrelation coefficient $K_{2}(\tau)$ also depend on the power law exponent. The reason why $K_{1}(\tau)$ or $K_{2}(\tau)$ have longer correlation time for smaller value of $R_{v}$ can be simply answered in the following way. Comparing the time series in fig. 5 a and b we

find that the motions are basically characterized by repeating the processes of starting from nearby zero, reaching the peak voltage and returning to zero again. As the maximum peak levels for $R_{v}=5 \Omega$ are roughly several times larger than the case of $R_{v}=150 \Omega$, each process of going up and down roughly takes several times longer time leading the longer correlation time.

\section{Conclusion}

We proposed an electrical analog circuit generating the fluctuation of which a probability density function has the power law tails. The circuit includes a noise generator, an analog multiplier and an operational amplifier for integrator. In our proposal circuit fluctuations with an arbitrary exponent of the power law can be obtained by tuning the value of resistance. We observed temporal outputs of the circuit and calculated the probability densitv 
the squared outputs. We show the probability density function with power law tails. The probability density function has different tails in positive and negative domains because the average of the additive noise is non-zero or its distribution function is asymmetrical. We conclude that the correlation time of the autocorrelation coefficient and that of the squared outputs depend on the value of resistance in the circuit. We expect that the proposed circuit is applicable to generate fluctuations having power law distribution in a much cheaper way than any digital computing methods. Moreover fluctuations of our circuit may be of use for risk estimation in foreign exchange or stock market in the near future.

\section{Acknowledgment}

One of the authors (A.-H. Sato) wishes to thank Yoshihiro Hayakawa for his stimulative discussion and his lecture on electrical circuits.

\section{References}

[1] H. Takayasu, A.-H. Sato and M. Takayasu, Phys. Rev. Lett., 79 (1997) 966.

[2] P. Jögi and D. Sornette and M. Blank, Phys. Rev. E, 57 (1998) 120.

[3] D. Sornette, Physica A, 250 (1998) 295.

[4] J. M. Deutsch, Physica A, 208 (1994) 433.

[5] S. C. Venkataramani, T. M. Antonsen Jr., E. Ott and J. C. Sommerer, Physica D, 96 (1996) 66.

[6] H. Nakao, Phys. Rev. E, 58 (1998) 1591.

[7] R. Benzi, G. Paladin, G. Parisi and A. Vulpiani, J. Phys. A, 18 (1985) 
[8] J. M. Deutsch, Phys. Rev. Lett., 69 (1992) 1536.

[9] Y. Kuramoto and H. Nakao, Phys. Rev. Lett., 76 (1996) 4352; 78 (1997) 4039 .

[10] A.-H. Sato, H. Takayasu, Physica A, 250 (1998) 231.

[11] A.-H. Sato, H. Takayasu and Y. Sawada, Phys. Rev. E, 61 (2000) 1081.

[12] M. Potters, R. Cont and J.-P. Bouchaud, Europhys. Lett., 41 (1998) 239.

Figure 1: Block diagram of all circuits with a noise generator. The circuit contains a noise generator, a analog multiplier and an operational amplifier for integrator. $R_{f}=100 \mathrm{k} \Omega, C=10 \mathrm{pF}, R_{v}=200 \Omega$. A variable resistor under the operational amplifier is for adjustment of the offset.

Figure 2: Semi-log plots of the probability density function of the multiplicative noise $\mu(t)$. Filled circles represent observation. A solid curve represents a Gaussian distribution with the same average and deviation as observation. From numerical estimation the average is 0.005103 , and the variance 0.006846 .

Figure 3: Autocorrelation coefficient of the multiplicative noise $\mu(t)$ for $125 \mathrm{kHz}$ sampling frequency. Filled circles represent observation. 
Figure 4: Semi-log plots of relation between a temporal average of the output $\langle v\rangle$ and the value of $R_{v}$. Filled and Unfilled circles represent observation with different offset voltage in the operational amplifier. A solid line represents $\langle v\rangle=4.5 \exp \left(-0.012 R_{v}\right)$. The fit of the data to exponential is good from 0 to $150 \Omega$.

Figure 5: Signal outputs for $R_{v}=5 \Omega$ (a) and $R_{v}=150 \Omega(\mathrm{b})$. The amplitude for $R_{v}=5 \Omega$ is larger than for $R_{v}=150 \Omega$.

Figure 6: Semi-log plots of probability density functions $R_{v}=5 \Omega$ (unfilled circles) and $R_{v}=150 \Omega$ (filled circles). The probability density function for $R_{v}=5 \Omega$ has wider tails than for $R_{v}=150 \Omega$.

Figure 7: Log-log plots of the probability density function for $R_{v}=5 \Omega$. Unfilled circles represent it in the positive domain and filled circles in the negative.

Figure 8: Log-log plots of $\mathrm{CDF}$ of the output $v_{o}(t)$ at $R_{v}=$ $5,25,50,100,150 \Omega$. CDFs at $R_{v}=50,100$ show straight lines with different slopes between a decade.

Figure 9: Log-log plots of autocorrelation coefficients for $R_{v}=5 \Omega$ (unfilled circle) and $R_{v}=150 \Omega$ (filled circle). The autocorrelation coefficient for $R_{v}=5 \Omega$ has a longer decay time than for $R_{v}=150 \Omega$.

Figure 10: Log-log plots of autocorrelation coefficients for squared output. 

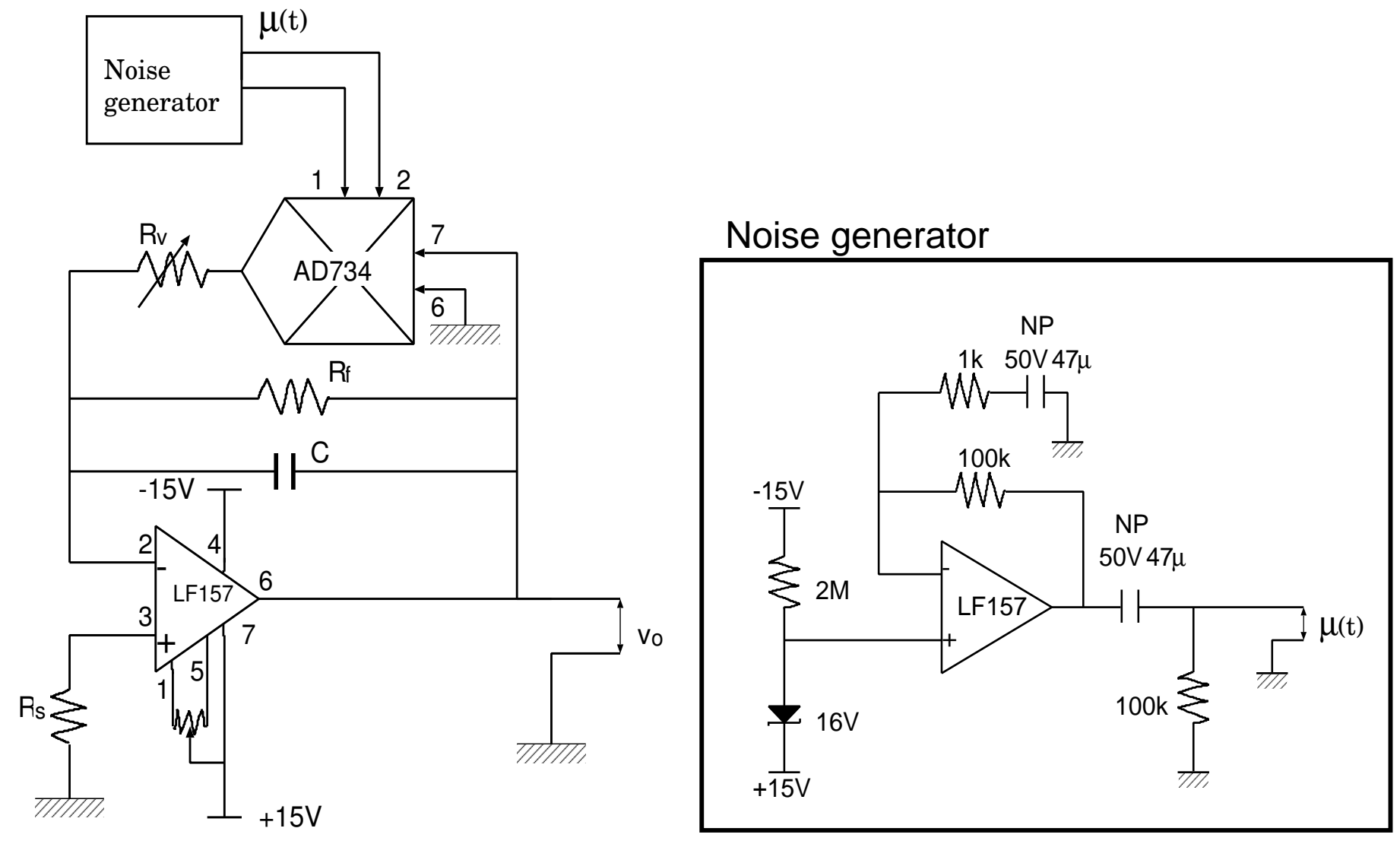


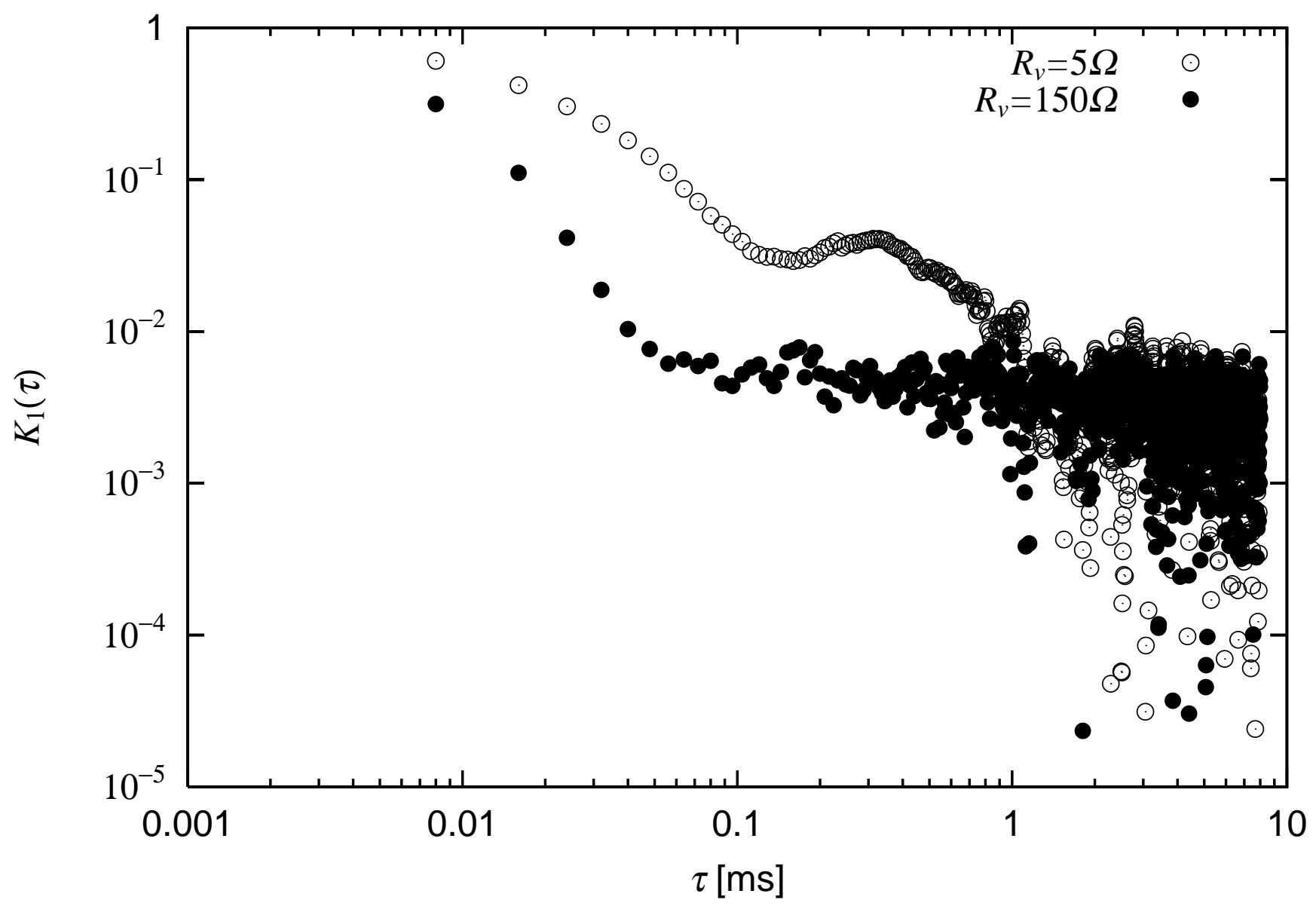




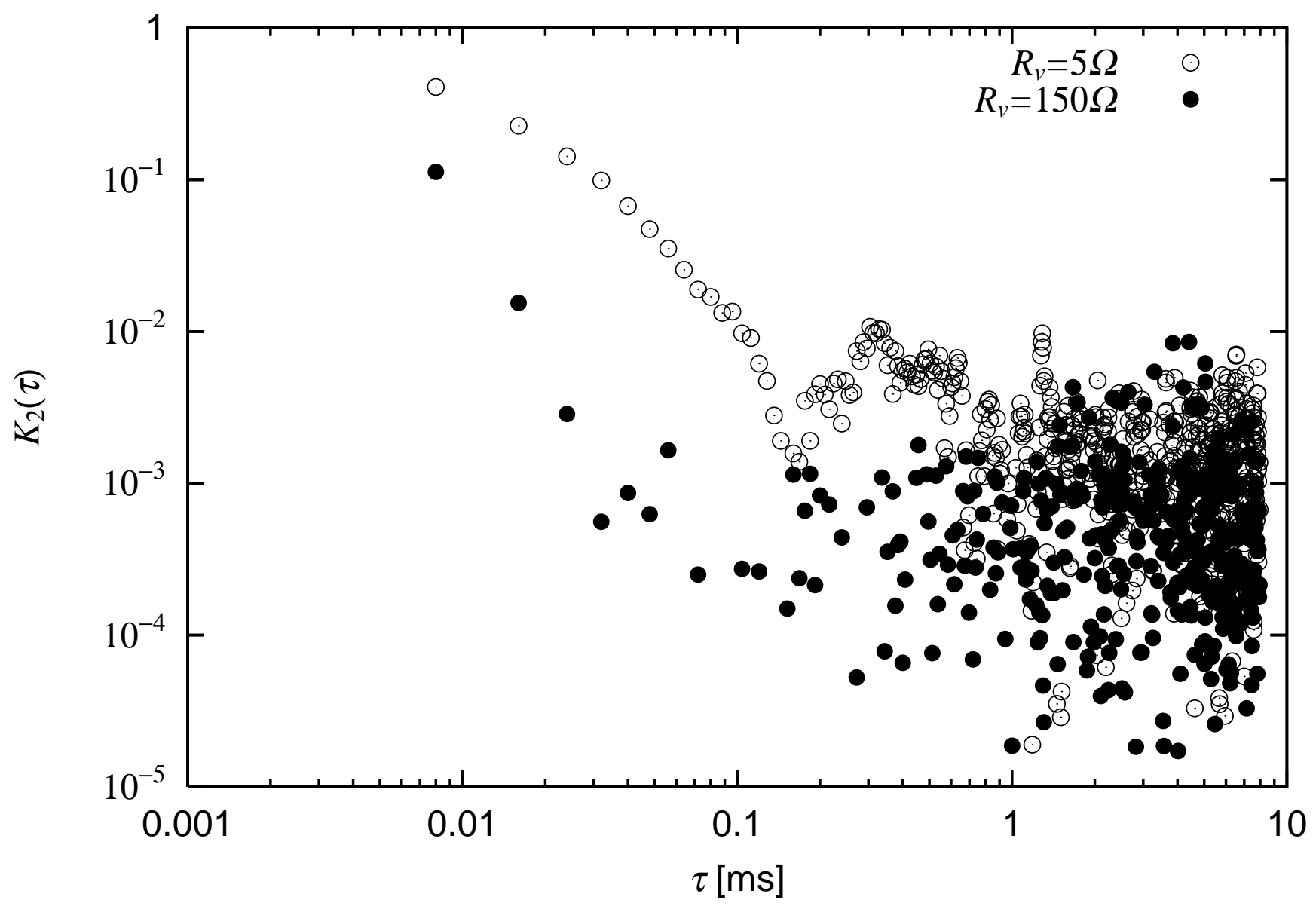




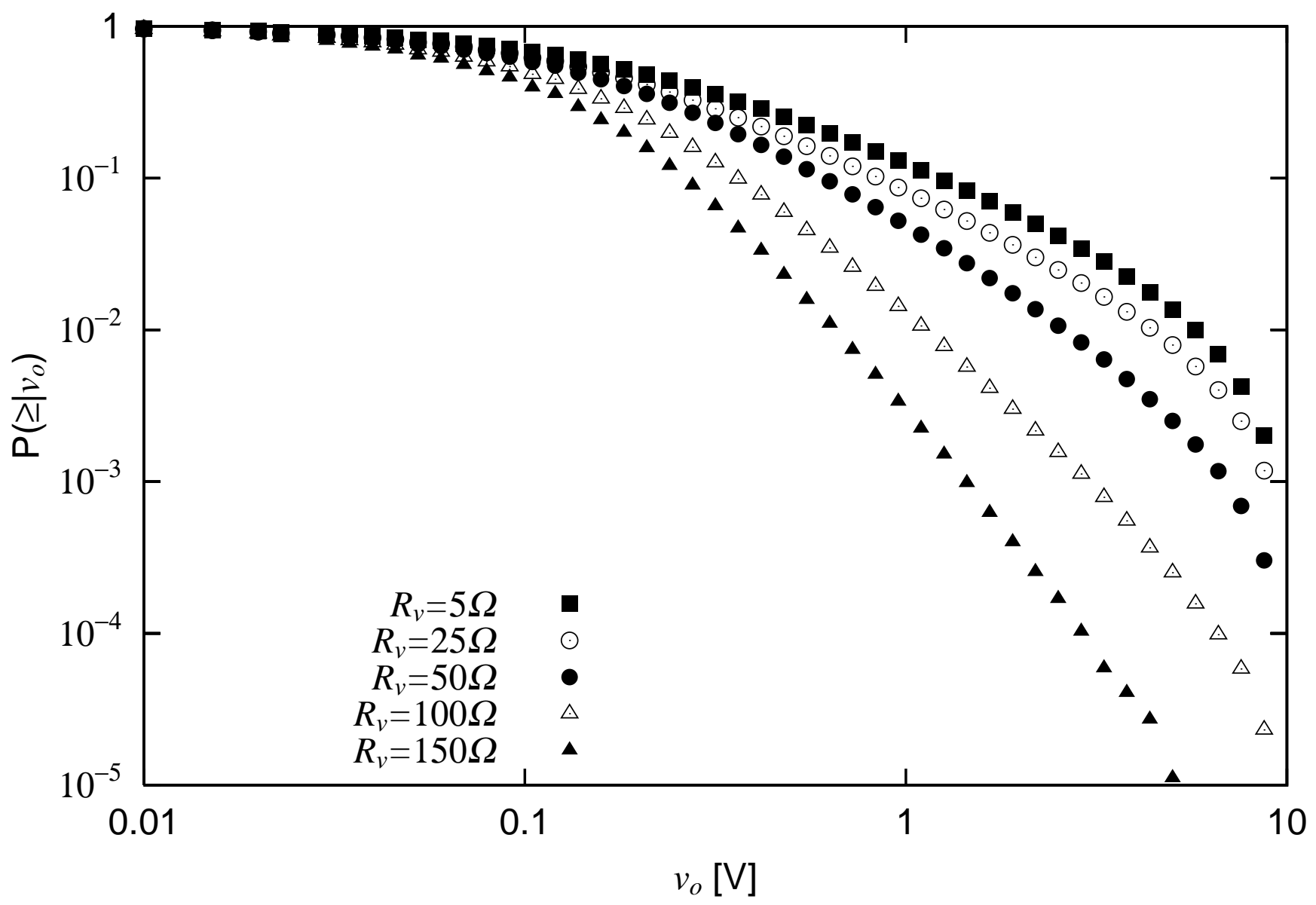




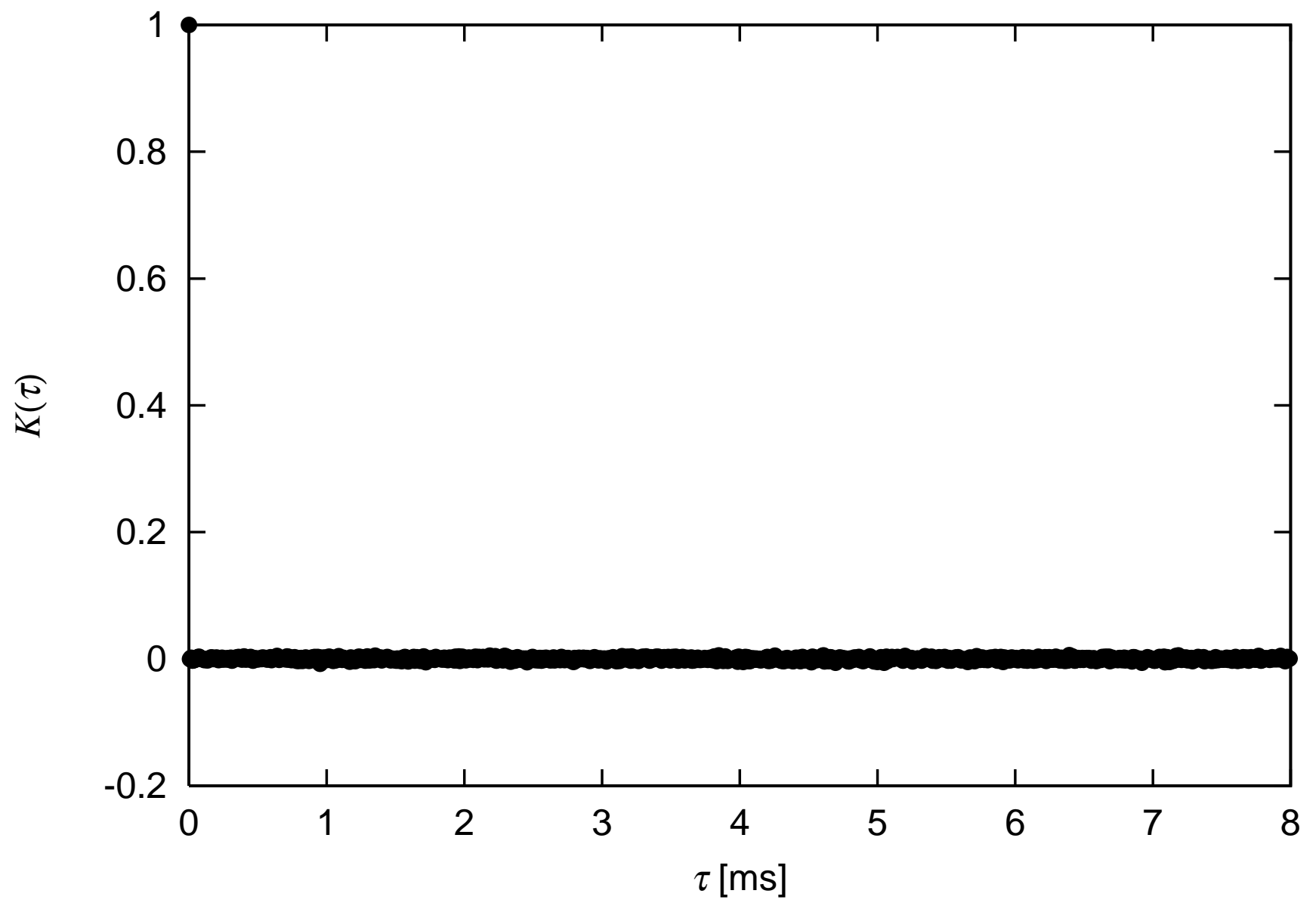




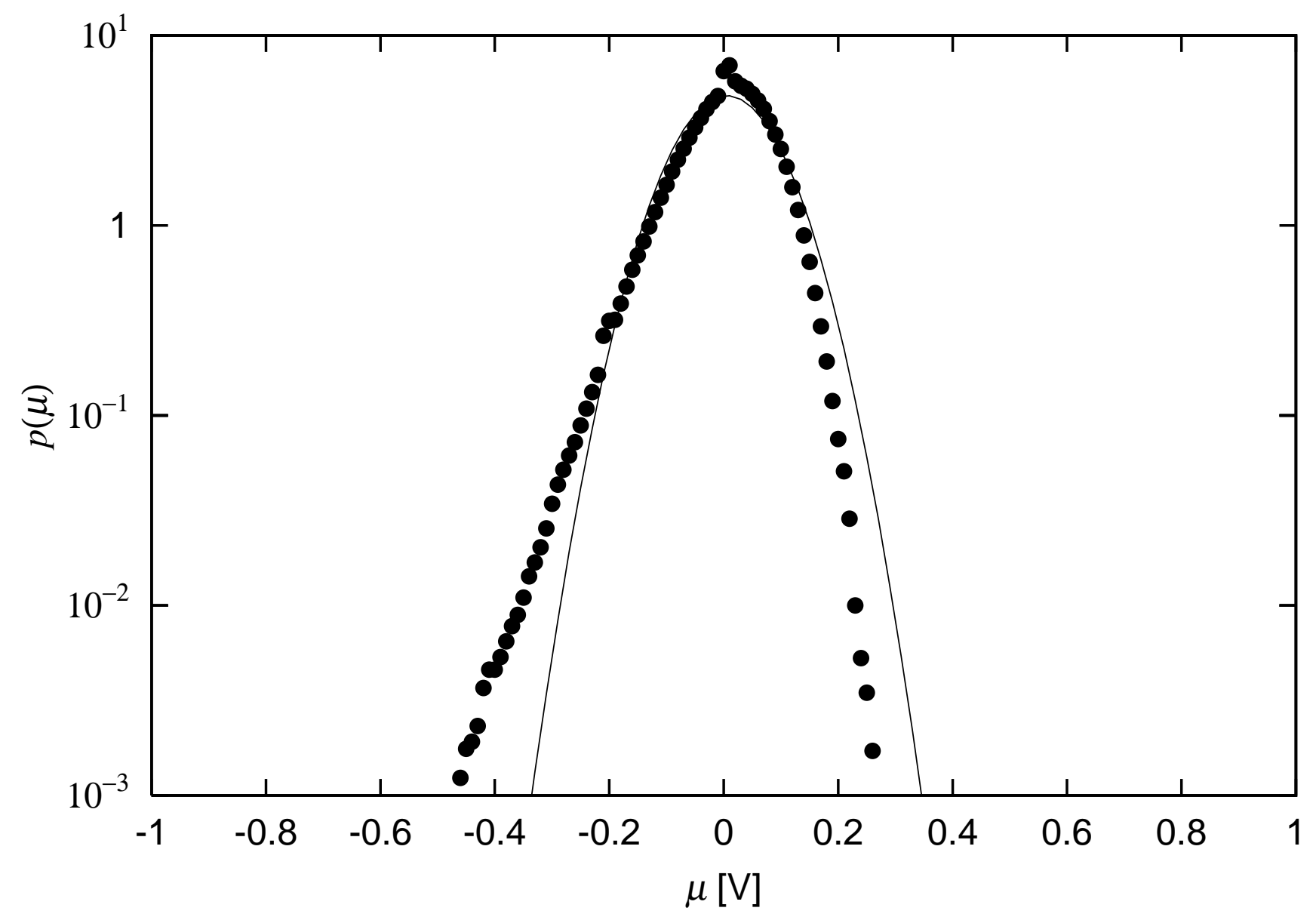




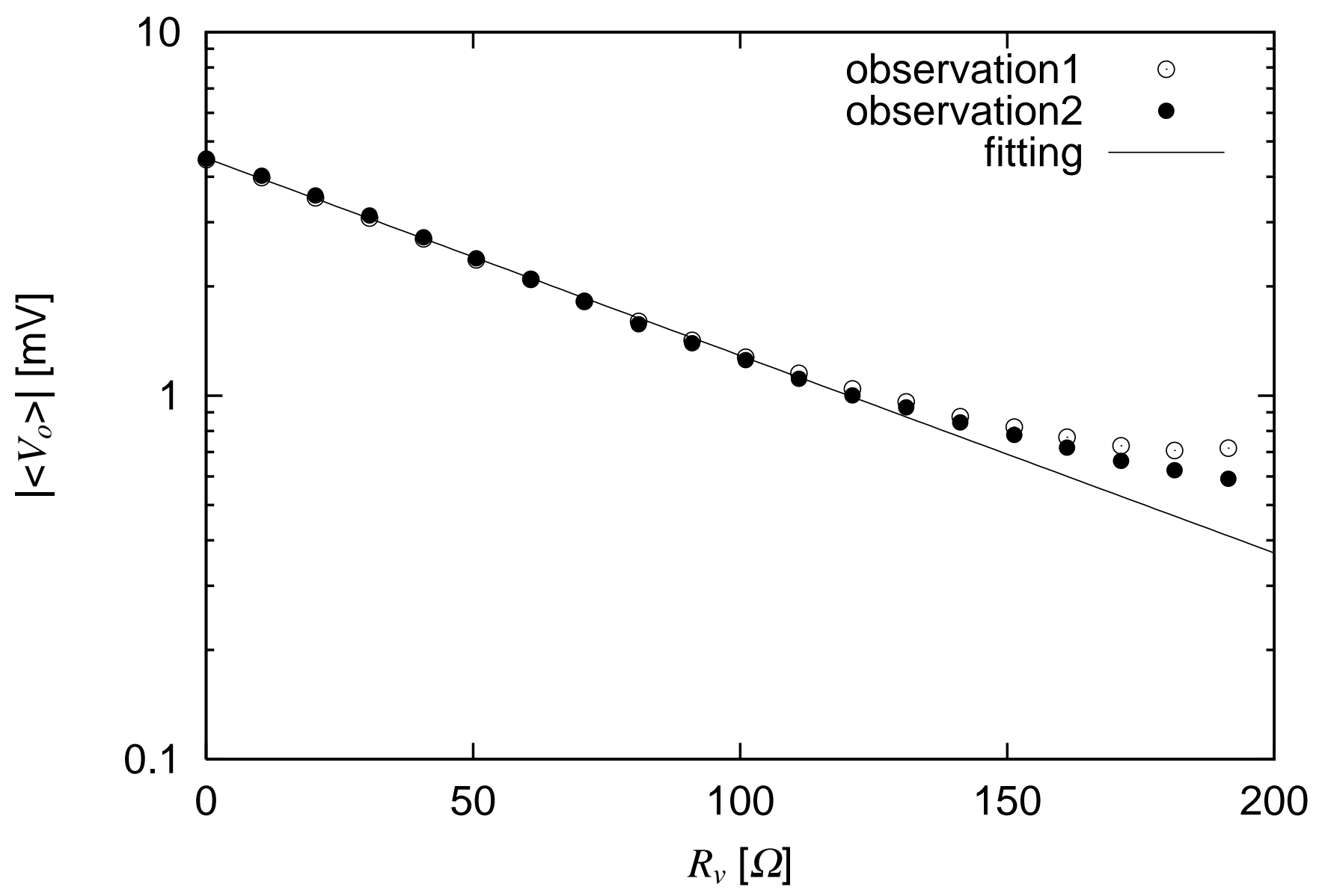




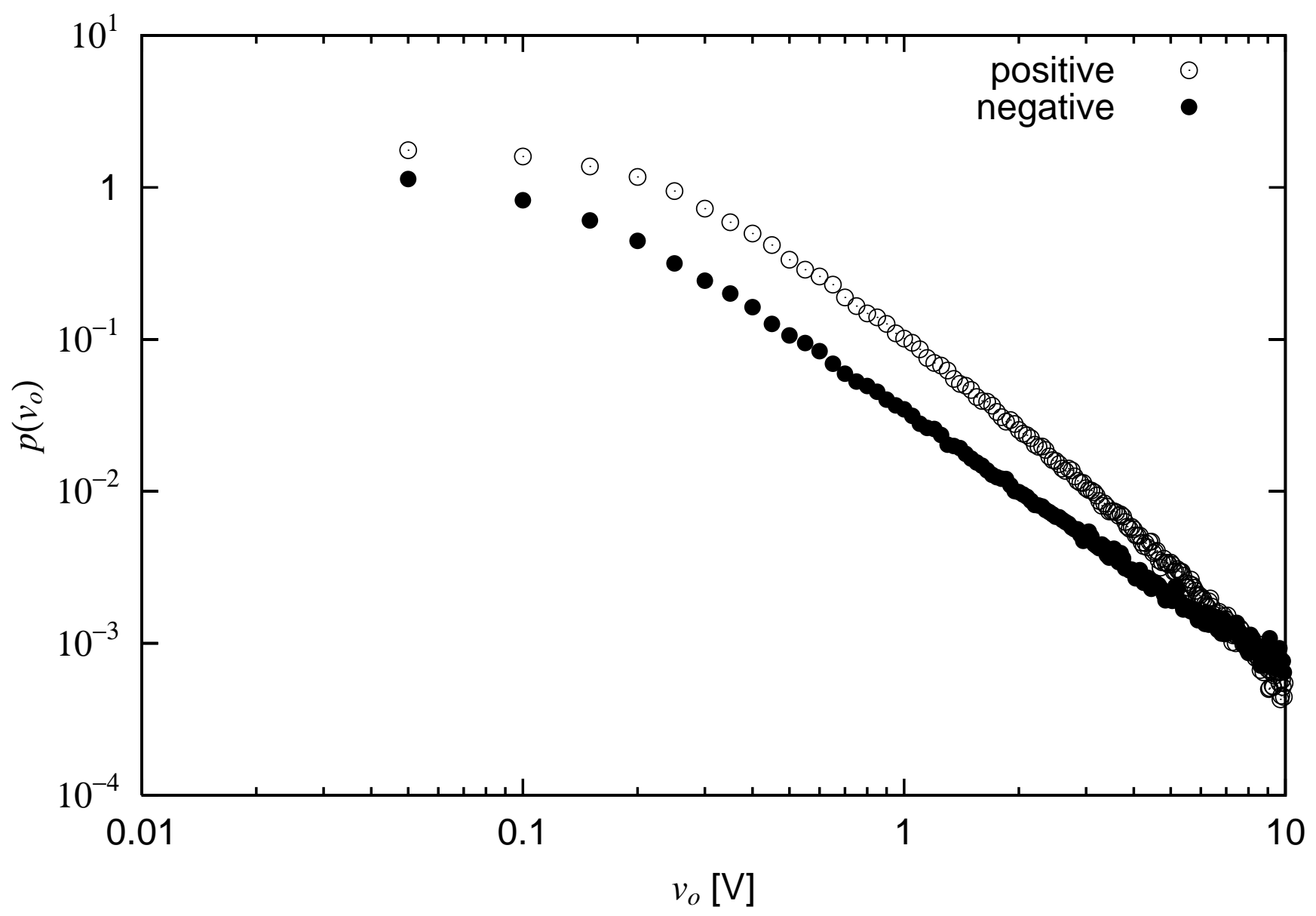




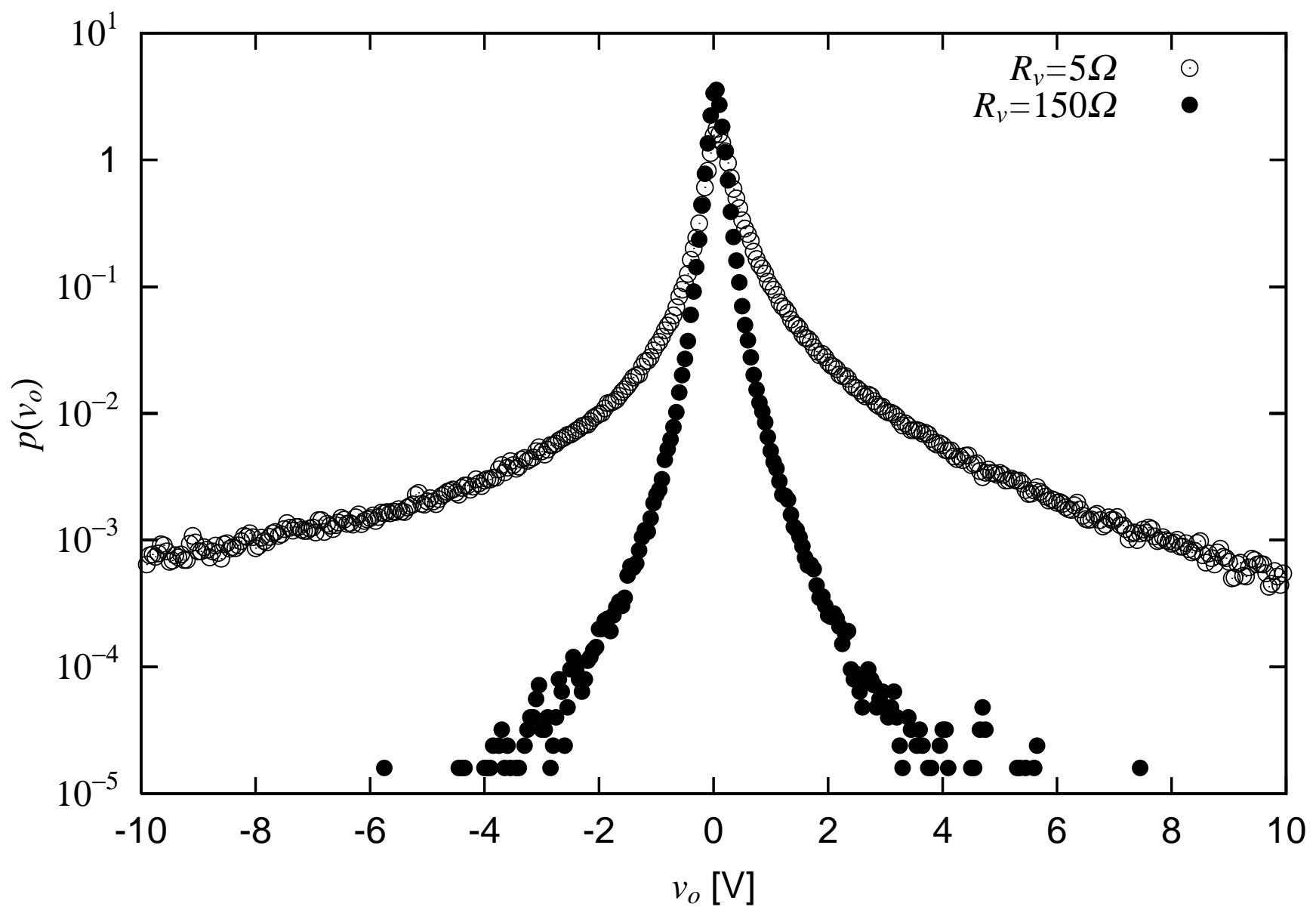




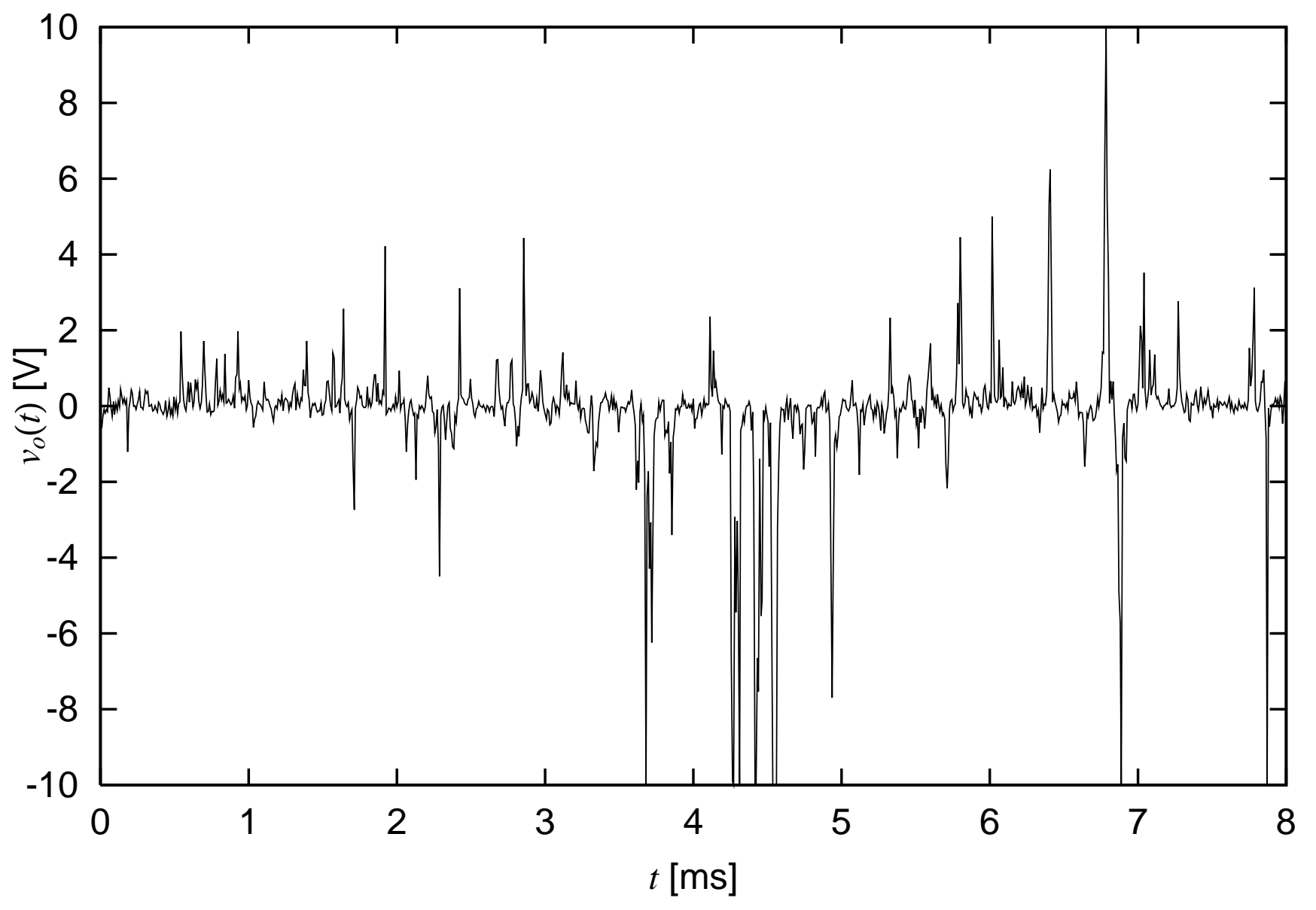




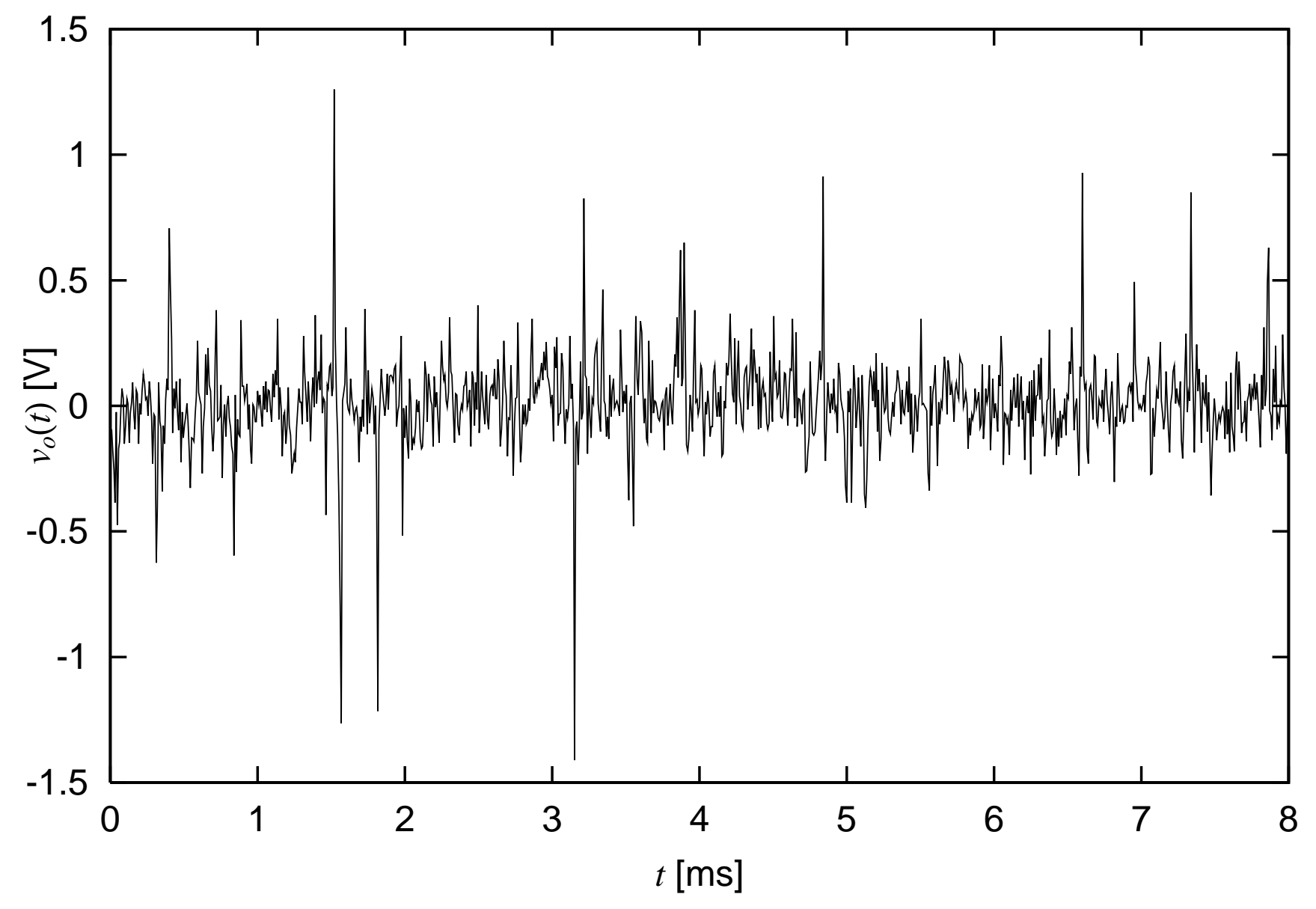

